\title{
Effects of ulinastatin on renal perfusion evaluated by Doppler ultrasonography in a porcine model of septic shock
}

\author{
CHEN-CHEN HANG ${ }^{1 *}$, YU-HONG GUO ${ }^{2 *}$, CHUN-SHENG LI $^{3}$ and SHUO WANG ${ }^{4}$ \\ ${ }^{1}$ Emergency Medicine Clinical Research Center of Beijing Chao-Yang Hospital, Capital Medical University, \\ Beijing Key Laboratory of Cardiopulmonary Cerebral Resuscitation, Beijing 100020; \\ ${ }^{2}$ Medical Affairs Office, Beijing Hospital of Traditional Chinese Medicine, Capital Medical University, \\ Beijing 100010; ${ }^{3}$ Department of Emergency Medicine, Beijing Friendship Hospital, \\ Capital Medical University, Beijing 100050; ${ }^{4}$ Department of Infectious Diseases (Fever Clinic), \\ Beijing Hospital of Traditional Chinese Medicine, Capital Medical University, Beijing 100010, P.R. China
}

Received June 17, 2021; Accepted August 19, 2021

DOI: $10.3892 /$ etm.2021.10759

\begin{abstract}
The present study aimed to evaluate the effect of ulinastatin (UTI) on renal perfusion using Doppler ultrasonography in a porcine model of septic shock induced by smoking inhalation and live methicillin-resistant Staphylococcus aureus instillation. A total of 32 healthy Landrace pigs were randomly assigned into the following four groups: Sham group ( $\mathrm{SH}$; $\mathrm{n}=5$ ), septic shock group ( $\mathrm{SS} ; \mathrm{n}=9$ ), septic shock treated with vancomycin $(15 \mathrm{mg} / \mathrm{kg})$ group (VAN; $\mathrm{n}=9)$ and septic shock treated with UTI $(50,000 \mathrm{U} / \mathrm{kg})+\operatorname{vancomycin}(\mathrm{UTI} ; \mathrm{n}=9)$ group. Renal perfusion was evaluated by contrast-enhanced ultrasound (CEUS) at baseline and at the end of the
\end{abstract}

Correspondence to: Dr Chun-Sheng Li, Department of Emergency Medicine, Beijing Friendship Hospital, Capital Medical University, 8 Yongan Road, Xicheng, Beijing 100050, P.R. China

E-mail: lichunsheng1962@126.com

Dr Shuo Wang, Department of Infectious Diseases (Fever Clinic), Beijing Hospital of Traditional Chinese Medicine, Capital Medical University, 23 Meishuguanhou Street, Dongcheng, Beijing 100010, P.R. China

E-mail: ysys2001@yeah.net

*Contributed equally

Abbreviations: AKI, acute renal injury; CO, cardiac output; CDFI, Color Doppler flow imaging; CEUS, contrast-enhanced ultrasound; ROI, regions of interest; UTI, ulinastatin; MRSA, methicillin-resistant Staphylococcus aureus; SVR, systemic vascular resistance; HR, heart rate; MAP, mean arterial pressure; Lac, lactic acid; cRI, corrected resistive index; SF6, sulphur hexafluoride; TIC, time-intensity curve; $\mathrm{Pi}$, peak intensity; AUC, area under curve; Th, time from peak to one half; LM, light microscope; EM, transmission electron microscope; AI, apoptotic index; GFR, glomerular filtration rate

Key words: acute kidney injury, septic shock, Doppler ultrasonography, contrast-enhanced ultrasound, ulinastatin protocol (24 h). The spectrum of interlobar or arcuate artery was selected to calculate the corrected resistive index (cRI). Sulphur hexafluoride microbubbles were bolus injected via a venous catheter. The peak intensity $(\mathrm{Pi})$ and area under curve (AUC) were calculated using a time-intensity curve. Compared with the baseline group, cRI was increased significantly at the end of the protocol, except for that in the SH group, whereas $\mathrm{Pi}$ decreased significantly after injury in all experimental groups but was higher in the UTI group compared with that in the SS and VAN groups (both $\mathrm{P}<0.001)$. Linear correlation was found between the cardiac output $(\mathrm{CO})$ and $\mathrm{Pi}\left(\mathrm{R}^{2}=0.752\right.$; $\mathrm{P}<0.001)$. The AUC was significantly decreased after injury in the SS and VAN groups compared with the baseline group. All parameters detected by CEUS were improved in the UTI group, and significant differences were found between the UTI and SS or VAN group (all $\mathrm{P}<0.05$ ). In conclusion, acute renal injury, which occasionally occurs during septic shock, is accompanied with a significantly lower perfusion rate in the renal microcirculation. By contrast, UTI can significantly improve renal perfusion, which can be reliably evaluated using CEUS.

\section{Introduction}

Multiple organ dysfunction induced by severe infection, sepsis and septic shock is one of the main causes of mortality in patients who are critically ill (1). Although treatment strategies have been gradually improving over the past decades, there remain to be obstacles regarding complications induced by pathophysiological processes, including acute renal injury (AKI). AKI occurs in $\sim 19 \%$ patients with moderate sepsis, $23 \%$ of patients with severe sepsis and $51 \%$ patients with septic shock when blood bacterial cultures are positive (1). The hemodynamic fluctuation during septic shock entails a phase of high cardiac output (CO) with low peripheral vascular resistance, followed by low $\mathrm{CO}$ with high peripheral resistance (2). The reaction to low $\mathrm{CO}$ in renal perfusion is different from other organs, which makes a more severe decrease in renal perfusion due to hypo-hemodynamic 
fluctuation than other organs when the patients suffer with sepsis (2).

Color Doppler flow imaging (CDFI) is a rapid, noninvasive and replicable technology for AKI detection, which has been used to assess changes in renal perfusion in patients who are critically ill $(3,4)$. In addition, contrast-enhanced ultrasound (CEUS) allows for a more accurate noninvasive estimation of the renal blood flow and provides quantitative measurements of local blood volume and velocity in selective regions of interest (ROI). Ulinastatin (UTI) is a human protease inhibitor that can be only isolated from the male urine because of the influence of estrogen, and it represents a glycoprotein with a typical Kuniz protease inhibitor structure $(5,6)$. UTI that has a broad spectrum of enzyme inhibitory activity $(5,6)$. Therefore, UTI has been used clinically for the prevention of multiple organ dysfunction $(7,8)$. However, there are few studies regarding its effect on AKI

The aim of the present study was to assess the potential effect of UTI on renal perfusion using Doppler ultrasonography in a porcine model of septic shock.

\section{Materials and methods}

Animal preparation. A total of 32 healthy male Landrace pigs aged 8-10 weeks and weighing $30 \pm 2 \mathrm{~kg}$, purchased from Lvyuanweiye Co. (license no. SCXK 11-00-002), were housed in cages at $20-25^{\circ} \mathrm{C}$ and humidity of $30-60 \%$ maintained by air conditioner with free access to food and water before the experiment. The room had a 12-h light/dark cycle. The present study was performed with the approval of the Ethics Committee of Beijing Chao-Yang Hospital, Capital Medical University (Beijing, China). Animal experiments were performed in accordance with the National Research Council's 1996 Guide for the Care and Use of Laboratory Animals (9).

Anesthesia was induced by midazolam $(0.2 \mathrm{mg} / \mathrm{kg}$, injected intramuscularly), followed by continuous intravenous infusion of pentobarbital $(8 \mathrm{mg} / \mathrm{kg} / \mathrm{h})$ and fentanyl $(5 \mu \mathrm{g} / \mathrm{kg} / \mathrm{h})$ for analgesia according to guidelines (9-12). Ringer's solution was administered intravenously to maintain sufficient preload. The right femoral artery and vein were dissected to insert a 5-Fr PiCCO catheter (Pulsiocath PV2015L20; Pulsion Medical Systems SE) into the descending aorta and to insert a central venous catheter (cat. no. CV-17702-E; Arrow International, Inc.) into the right atrium. The arterial and central venous catheters were connected to a monitor (Philips Medical Systems B.V.) to continuously monitor the hemodynamics and blood temperature.

After anesthesia, all animals were intubated using a cuffed 6.5-mm endotracheal tube. Mechanical ventilation was provided by a ventilator (Evita 4; Dräger Medizintechnik) with a tidal volume of $8 \mathrm{ml} / \mathrm{kg}$, a respiratory frequency of $12 \mathrm{breaths} / \mathrm{min}$ and a positive end-expiratory pressure of $5 \mathrm{~cm} \mathrm{H} \mathrm{H}_{2} \mathrm{O}$. The arterial oxygen saturation $\left(\mathrm{SaO}_{2}\right)$ and end tidal concentration of $\mathrm{CO}_{2}\left(\mathrm{EtPCO}_{2}\right)$ were continuously measured by the monitor. The respiratory frequency and the fraction of inspired oxygen $\left(\mathrm{FiO}_{2}\right)$ were adjusted to maintain an $\mathrm{EtPCO}_{2}$ of $35-40 \mathrm{mmHg}$ and an $\mathrm{SaO}_{2}$ of $>90 \%$.

Experimental protocols. Baseline data were obtained after $1 \mathrm{~h}$ of model establishment. Animals were randomly assigned to the following four groups: i) The sham group ( $\mathrm{SH}$; n=5); ii) the septic shock group (SS; n=9); iii) the septic shock treated with vancomycin group (VAN; $500 \mathrm{mg}$; Eli Lilly and Company; $\mathrm{n}=9)$; and iv) the septic shock treated with UTI (50,000 U; Guangdong Techpool Bio-Pharma Co. Ltd.) + vancomycin group (UTI; $n=9$ ). The number of animals was confirmed according to a previous investigation (12). No other procedures were performed in the $\mathrm{SH}$ group apart from catheter insertion and mechanical ventilation. All animals in the three experimental groups were exposed to smoke from $50 \mathrm{~g}$ burning cotton using a bee smoker according to previously described methods (13-15). Arterial carboxyhemoglobin levels were measured using a portable monitor (Rad-57; Masimo Corp.) to ensure that each animal had received an equivalent dose of smoke. After 12 breaths of cotton smoke were insufflated into the lungs, animals were allowed to breathe normal air for 2 min. Four sets of smoke inhalations (12 breaths of smoke, 2 min rest, 12 breaths of smoke; total of 48 breaths) were performed.

Live methicillin-resistant Staphylococcus aureus (MRSA), which was separated from the blood of a middle-aged male patient with blood infection (12), was cultured at the bacterial experimental lab of Chao-Yang Hospital (Beijing, China). Following smoking injury, $3 \times 10^{11} \mathrm{cfu}$ MRSA suspended in $30 \mathrm{ml}$ sterile saline were instilled into the lungs of animals except for the sham group using a bronchoscope (12). Subsequently, $15 \mathrm{mg} / \mathrm{kg}$ vancomycin or $15 \mathrm{mg} / \mathrm{kg}$ of vancomycin $+50,000 \mathrm{U} / \mathrm{kg}$ UTI dissolved in $100 \mathrm{ml}$ saline were infused into the central venous catheter every $12 \mathrm{~h}$ in the VAN and UTI group, respectively. Before the humane endpoints of the study, $10 \mathrm{ml}$ blood were sampled into an aerobic culture bottle (BacT/ALERT FA; bioMérieux Inc.) for blood culture.

All animals were continuously monitored during the entire protocol. The humane endpoints of the study were $24 \mathrm{~h}$ after modeling or no pulse signal detected on the monitor. After that, euthanasia was performed by the infusion of overdose pentobarbital $(40 \mathrm{mg} / \mathrm{kg})$ followed by a lethal dose potassium chloride $(15 \% \mathrm{KCl} 500 \mathrm{mg} / \mathrm{kg})$ in accordance with the AVMA guidelines for the euthanasia of animals $(16,17)$. Death was confirmed by the cessation of vital signs, which were shown as the single lines of pulse and electrocardiograph on the monitor. The right kidney was then dissected for histopathology testing. The diagram of experimentation is presented in Fig. 1.

Hemodynamics. A total of $10 \mathrm{ml}$ of $4^{\circ} \mathrm{C}$ saline was injected into the right atrium through the central venous catheter at 0 (baseline), 2, 6, 12 and $24 \mathrm{~h}$ to determine $\mathrm{CO}$ using the thermodilution method (18). Systemic vascular resistance (SVR) was calculated by the monitor. All animals were anaesthetized continuously for $24 \mathrm{~h}$. Heart rate (HR) and mean arterial pressure (MAP) were recorded continuously.

A total of $1 \mathrm{ml}$ arterial blood samples for blood gas and lactic acid (Lac) analysis (GEM Premier 3000 Blood Gas Analyzer; Instrumentation Laboratory) were collected from the PiCCO catheter at the baseline and $24 \mathrm{~h}$ after injury.

CDFI and CEUS. CDFI and CEUS were performed on the right kidney at the baseline and the end of the protocol (24 h) by an experienced ultrasound physician who was blinded to the study, using an ultrasound system (Aplio 500 TUS-A500; 


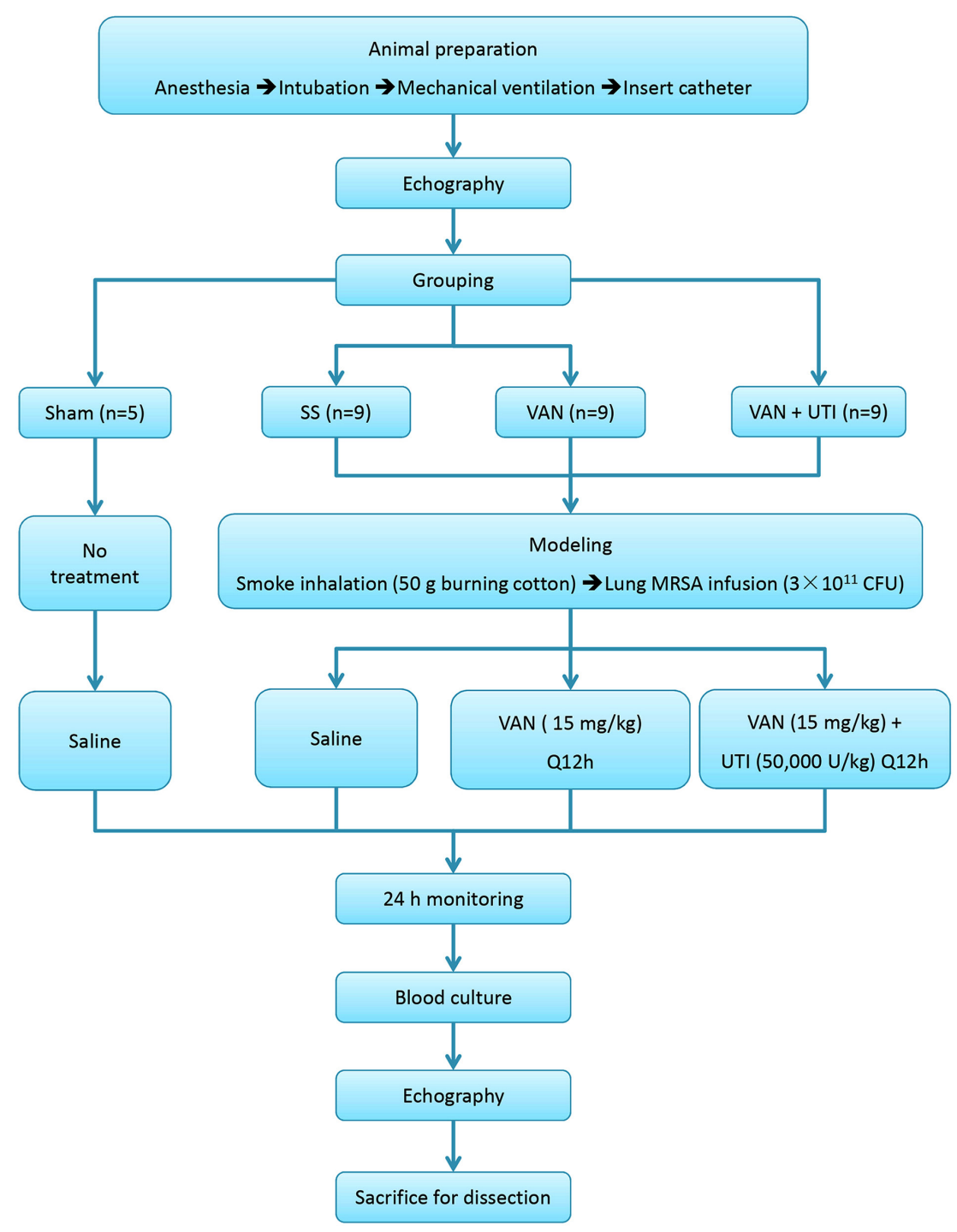

Figure 1. Schematic representation of the protocol in the present study. SS, septic shock group; UTI, ulinastatin group; VAN, vancomycin group; MRSA, methicillin-resistant Staphylococcus aureus.

Canon Medical Systems Corporation). The probe was put on the right side of the animal's abdomen to obtain a clear image of the right kidney. An interlobar or arcuate artery was selected to obtain result from the measurement. The spectrum was considered optimal when $\geq$ three similar consecutive waveforms were visualized (Fig. 2A-D). The corrected resistive index (cRI) was calculated using the following equations (3). Three measurements were performed and averaged to obtain the mean $\mathrm{cRI}$ value. $\mathrm{RI}=$ (peak systolic velocity-minimum diastolic velocity)/peak systolic velocity. cRI=[observed RI -0.0026 x (80-observed HR)].

For CEUS, $2 \mathrm{ml}$ sulphur hexafluoride $\left(\mathrm{SF}_{6}\right)$ microbubbles $\left(8 \mu 1 \mathrm{SF}_{6} / \mathrm{ml}\right.$; SonoVue ${ }^{\oplus} ;$ Bracco Suisse SA) were bolus injected via the central venous catheter. ROI $\left(26 \mathrm{~mm}^{2}\right)$ was manually drawn within the renal cortex to create a time-intensity curve (TIC) with the QLAB software (Philips Healthcare). The peak intensity $(\mathrm{Pi})$, area under the curve (AUC), time from peak to one half (Th), time to peak (Tp) and wash-in slope (Slope) were calculated by the QLAB software automatically (Fig. 3A-D and Videos S1-4). Three injections were done at intervals of $5 \mathrm{~min}$ to obtain an average value. The same renal cortex ROI was used to prevent the influence of the respiratory motion for each bolus injection.

Pathology and apoptosis assay. After the animals were euthanized, the upper pole of right renal cortex was surgically removed and preserved in $4 \%$ paraformaldehyde $>24 \mathrm{~h}$ at room temperature. For light microscope (LM) observation, paraffin embedded tissues were cut as a $10 \mu \mathrm{m}$ thickness. After dewaxing and rehydration, H\&E staining were performed at room temperature for $10 \mathrm{~min}$. Dehydrated slices were sealed by neutral gum and covered with glass. Subsequently, a grade of renal injury of semi-quantitative evaluation was performed in each animal (Table I) under LM at $\mathrm{x} 400$ magnification according to previous study (19). TUNEL assay was used to label cells in tissues that suffered severe DNA damage/fragmentation induced by apoptotic signaling cascade activation. The prepared $10 \mu \mathrm{m}$ sections were incubated for $60 \mathrm{~min}$ at $37^{\circ} \mathrm{C}$ with TUNEL reagent according to the manufacturer's protocol (cat. no. C1091; Beyotime Institute of Biotechnology). After hematoxylin counterstain for $10 \mathrm{sec}$ at room temperature, TUNEL-positive cells, which 


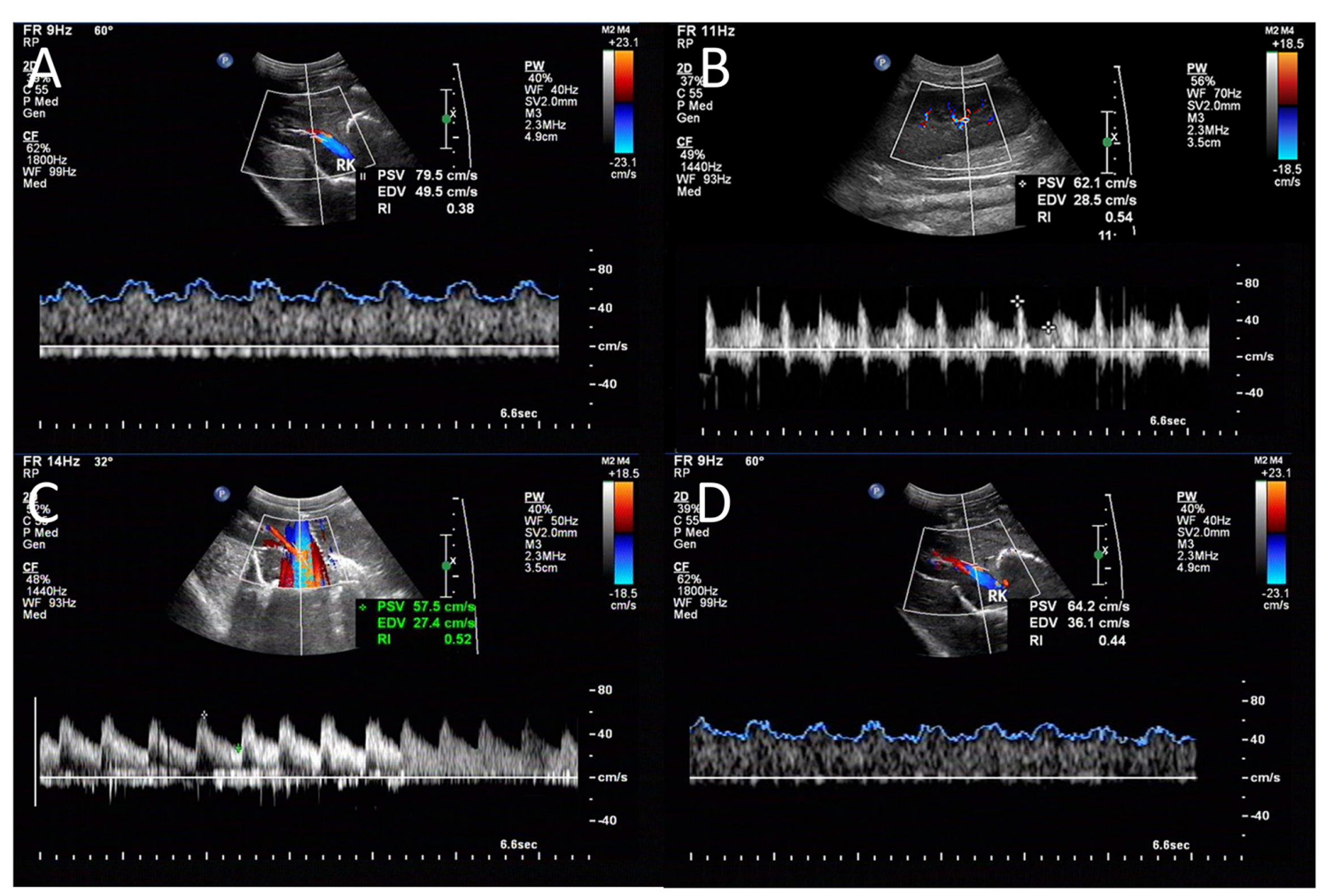

Figure 2. Representative color Doppler flow and contrast enhanced ultrasound images. Doppler gain was set in order to obtain a clear outline of flow waves with minimal background noise. An interlobar or arcuate artery was selected and the results were displayed automatically on the screen. (A) SH group. (B) SS group. (C) VAN group. (D) UTI group. SH, sham group; SS, septic shock group; VAN, vancomycin group; UTI, the ulinastatin group; ROI, region of interest.

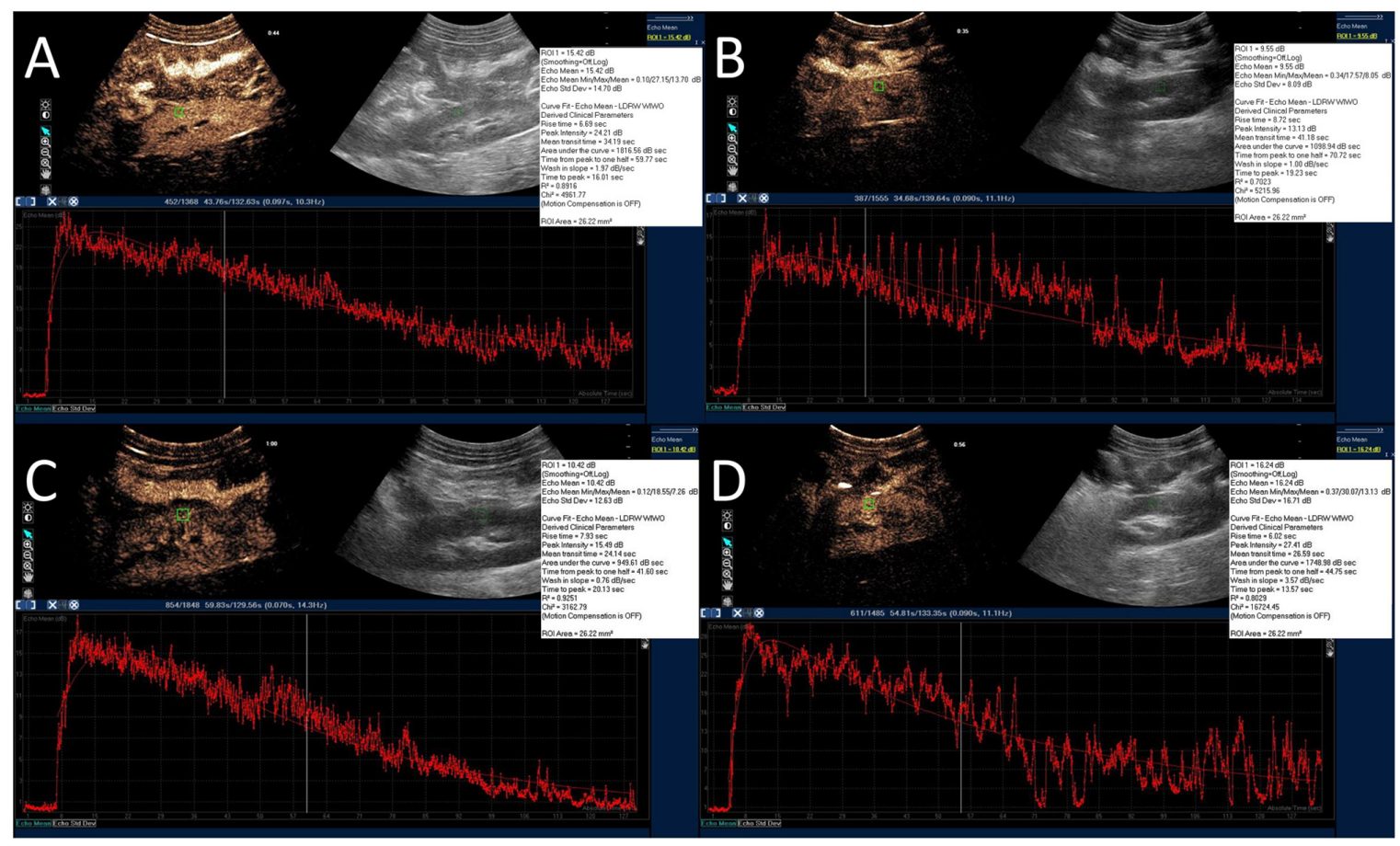

Figure 3. Representative CEUS images. For CEUS, $2 \mathrm{ml}$ sulphur hexafluoride microbubbles were bolus injected via the central venous catheter. A ROI $\left(26 \mathrm{~mm}^{2}\right.$; green square) was manually drawn within the renal cortex to create a time-intensity curve with the ultrasound system's QLAB software (red curves). The peak intensity, area under the curve, time from peak to $50 \%$ of maximum, time to peak and wash-in slope were calculated by the software automatically. (A) SH group. (B) SS group. (C) VAN group. (D) UTI group. CEUS, contrast enhanced ultrasound; SH, sham group; SS, septic shock group; VAN, vancomycin group; UTI, the ulinastatin group; ROI, region of interest.

were colored brown, were counted to determine the apoptotic index (AI) under LM at $\mathrm{x} 400$ magnification. $\mathrm{AI}=$ apoptotic cells staining brown/total TUNEL-positive cells x100\%. The pathological evaluations were performed by an independent 
Table I. Pathologic change for a semi-quantitative evaluation of kidney injury.

Grade

Pathological changes under light microscope

\section{0, Normal Normal renal histopathology}

1, Mild Mild interstitial edema, renal tubular necrosis, slight glomerular capillary angiectasis and inflammatory cell infiltration

2, Moderate Pathologic changes of renal between mild and severe

3, Severe Severe interstitial edema, renal tubular necrosis, severe glomerular capillary angiectasis, mesangial proliferation and severe inflammatory cell infiltration

A
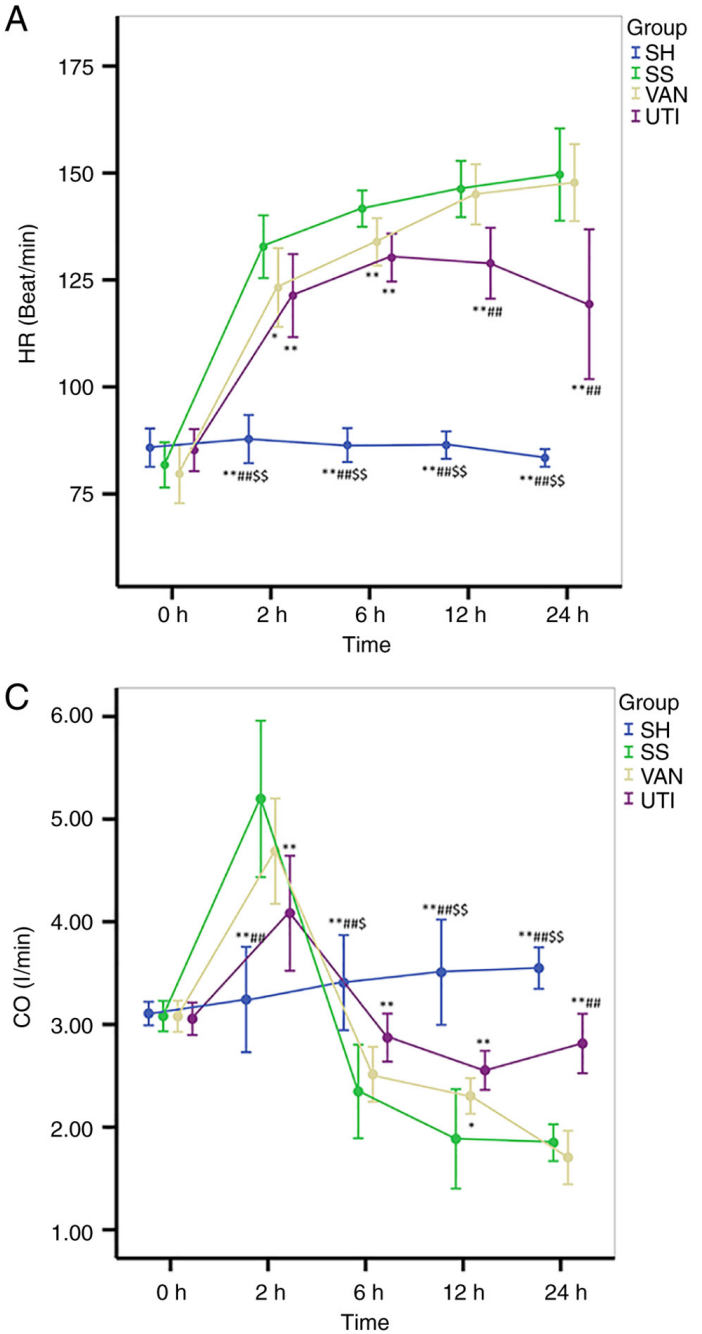

$B$
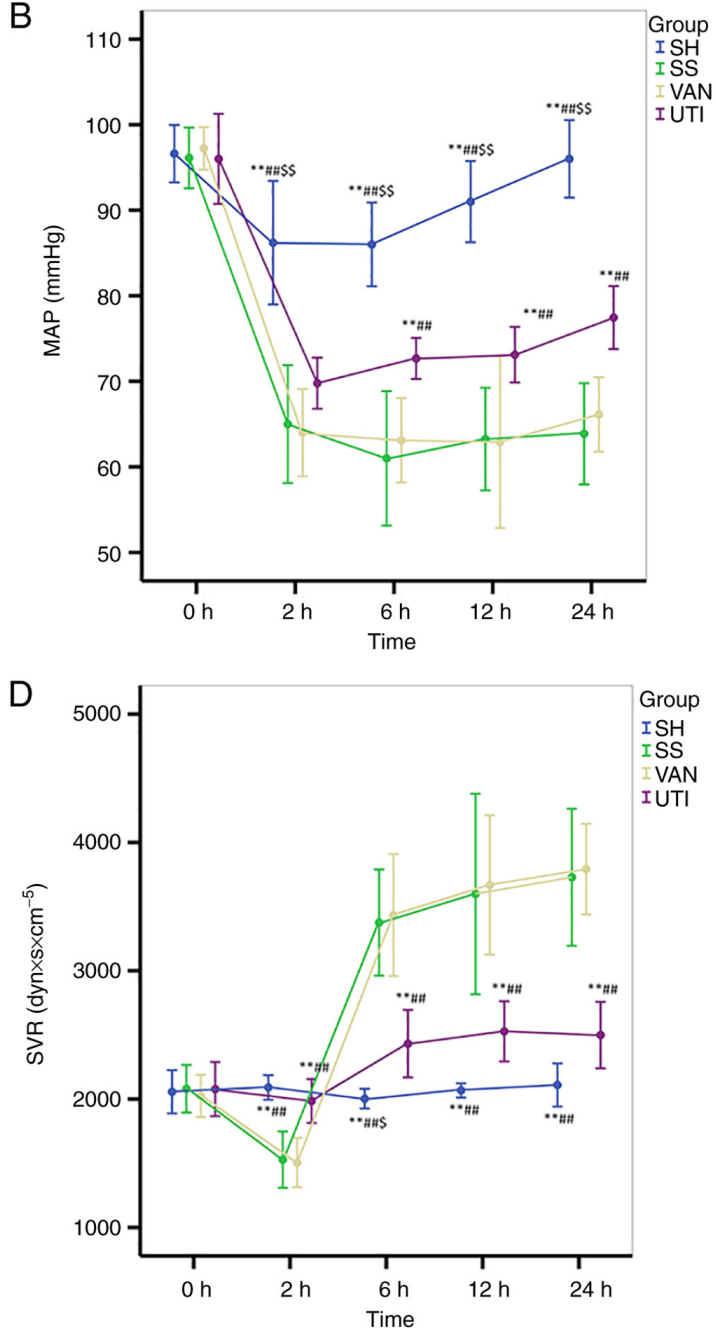

Figure 4. Hemodynamics. All parameters in the SH group remained at a relatively normal level throughout the entire protocol. The changes in the VAN group were similar to those in the SS group. (A) In the UTI group, HR decreased slowly $12 \mathrm{~h}$ after injury. (B) In the SS and VAN group, MAP decreased to 60-70 mmHg $2 \mathrm{~h}$ after injury, which remained at a low level. In the UTI group, MAP was improved progressively $6 \mathrm{~h}$ after injury. Changes in the (C) CO and (D) SVR in the SS and VAN groups were in the opposite direction: CO increased $2 \mathrm{~h}$ after injury and then decreased, whereas SVR decreased at the same time point then increased. The fluctuations in CO and SVR in the UTI group were ameliorated. ${ }^{*} \mathrm{P}<0.05,{ }^{* *} \mathrm{P}<0.01$ vs. the SS group; ${ }^{\# \prime} \mathrm{P}<0.01$ vs. the VAN group; ${ }^{\$} \mathrm{P}<0.05,{ }^{\$} \mathrm{P}<0.01$ vs. the UTI group. HR, heart rate; MAP, mean arterial pressure; CO, cardiac output; SVR, systemic vascular resistance. SH, sham group; SS, septic shock group; VAN, vancomycin group; UTI, ulinastatin group.

pathologist who has $>10$ years of experience and was blinded to the present study.

Another part of the specimens was double fixed in $2.5 \%$ glutaraldehyde solution at $4^{\circ} \mathrm{C}$ for $4 \mathrm{~h}$ with Millonig's phosphate buffer $(\mathrm{pH}=7.3)$. The samples were incubated for $1 \mathrm{~h}$ in $1 \%$ osmium tetroxide, then placed into $1: 1 \mathrm{mix}$ of acetone and epoxy resin for $12 \mathrm{~h}$ and $100 \%$ epoxy resin to polymerize overnight at $37^{\circ} \mathrm{C}$. For the solidifying process, the samples were left to polymerize at $37^{\circ} \mathrm{C}, 60^{\circ} \mathrm{C}$ and $72^{\circ} \mathrm{C}$ for $24 \mathrm{~h}$ respectively, then $50-100 \mathrm{~nm}$ ultrathin sections of the specimens were obtained. After 3\% uranyl acetate and 3\% lead nitrate double staining for $30 \mathrm{~min}$, the specimens were 

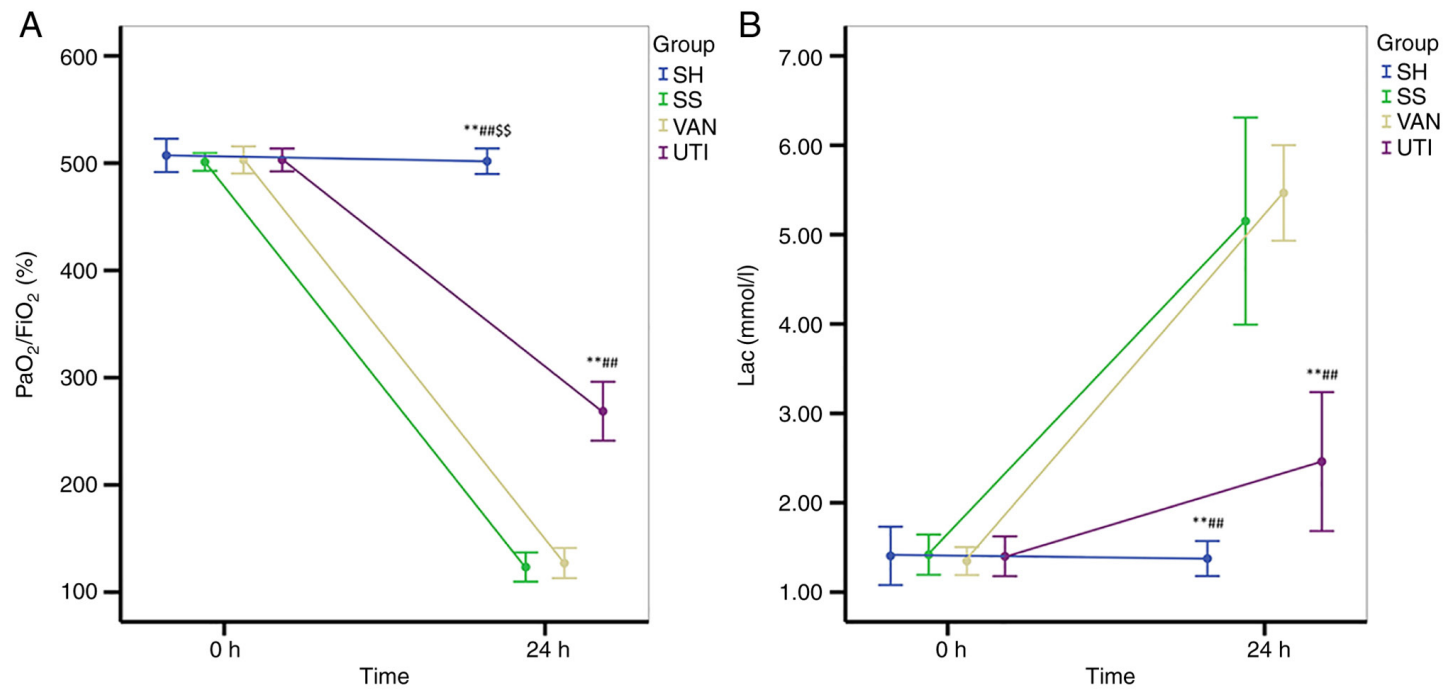

Figure 5. Oxygenation and Lac. (A) A total of $24 \mathrm{~h}$ after the baseline, the $\mathrm{PaO}_{2} / \mathrm{FiO}_{2}$ ratio in the UTI group was higher than the SS and VAN group but lower than that in the SH group. (B) On the contrary, the Lac concentration in the UTI group was lower than the SS and VAN group. ** P $<0.01$ vs. the SS

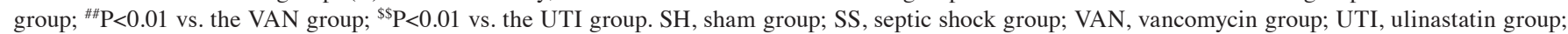
$\mathrm{PaO}_{2}$, partial pressure of arterial oxygen; $\mathrm{FiO}_{2}$, fraction of inspired oxygen; Lac, lactic acid.

observed under a transmission electron microscope (TEM; HT7700; Hitachi, Ltd.).

Statistical analysis. Statistical analysis was performed on SPSS 19.0 software (IBM Corp.). Continuous variables with normal distribution were confirmed using Kolmogorov-Smirnov test, were presented as the mean $\pm \mathrm{SD}$. The mixed two-way ANOVA was used to analyze the effect of time as a repeated measure and treatment groups as a between-subjects factor. Subsequent analyzes of specific differences at individual time points were performed with Bonferroni test. Ordinal data (grade of renal injury) were presented as median (IQR) and compared with Kruskal-Wallis followed by Dunn's test. Linear regression was performed to determine associations between the parameters. $\mathrm{P}<0.05$ was considered to indicate a statistically significant difference.

\section{Results}

Outcomes. No significant differences in blood temperature at baseline were found among the four groups $(\mathrm{P}=0.954)$. A rapid increase in blood temperature to $>40^{\circ} \mathrm{C}$ was observed in the three experimental groups within $2 \mathrm{~h}$ after injury, whilst it remained normal in the SH group during the entire protocol. In total, one animal in the SS and VAN groups died at $16 \mathrm{~h}$ and $20 \mathrm{~h}$ after injury, respectively. All other animals remained alive until the end of protocol. All blood cultures of the $\mathrm{SH}$, VAN and UTI group were tested negative, whereas six of the nine animals in the SS group were tested positive for MRSA (data not shown).

Hemodynamics and oxygenation. All parameters, including HR, MAP, CO and SVR, in the SH group stayed at a relatively normal level throughout the entire protocol. HR increased significantly in the SS group compared with the SH and UTI group, which was higher than $133 \pm 7$ beats/min $2 \mathrm{~h}$ after injury. The changes in HR in the VAN group were similar to those in the SS group. However, HR decreased slowly $12 \mathrm{~h}$ after injury in the UTI group, with significant differences compared with that in the SS and VAN groups (Fig. 4A).

In the SS and VAN groups, MAP decreased to 60-70 $\mathrm{mmHg} 2 \mathrm{~h}$ after injury, which remained low thereafter. MAP in the UTI group was also decreased $2 \mathrm{~h}$ after injury, but improved progressively thereafter, with significantly higher values in comparison with the SS or VAN groups at each time point from $6 \mathrm{~h}$ after injury onwards (Fig. 4B). Opposite trends in the changes in CO and SVR in both SS and VAN groups were found. $\mathrm{CO}$ increased $2 \mathrm{~h}$ after injury and then decreased (Fig. 4C). By contrast, the SVR decreased at $2 \mathrm{~h}$ and then increased (Fig. 4D). The magnitude of the fluctuation in CO in the UTI group were not as large as that in the SS and VAN groups (Fig. 4C). In addition, at $24 \mathrm{~h}$ after injury, $\mathrm{CO}$ was significantly higher in the UTI group compared with that in the SS or VAN group (Fig. 4C). SVR in the UTI group increased slightly at $6 \mathrm{~h}$ after injury, then remained stable at the level of slightly higher than that in the SH group thereafter (Fig. 4D). However, it was significantly lower compared with that in the SS or VAN group (Fig. 4D).

In the SS and VAN groups, the ratio of the partial pressure of arterial oxygen $\left(\mathrm{PaO}_{2}\right)$ to $\mathrm{FiO}_{2}$ was decreased after modeling, which remained at $<200 \%$ (Fig. 5A). The $\mathrm{PaO}_{2} / \mathrm{FiO}_{2}$ ratio in the UTI group was higher compared with that in the SS and VAN groups, but lower than that in the SH group (Fig. 5A). Lac levels were significantly increased in all three of the experimental groups compared with those in the $\mathrm{SH}$ group, but lac levels were lower in the UTI group compared with those in the SS and VAN groups (Fig. 5B).

CDFI and CEUS results. No significant difference of cRI was observed at the baseline among all four groups, but cRi increased significantly at $24 \mathrm{~h}$ compared with the baseline except for that in the SH group (Fig. 6A). However, a significant difference of cRI was found between each experimental group and that in the SH group (Fig. 6A). 

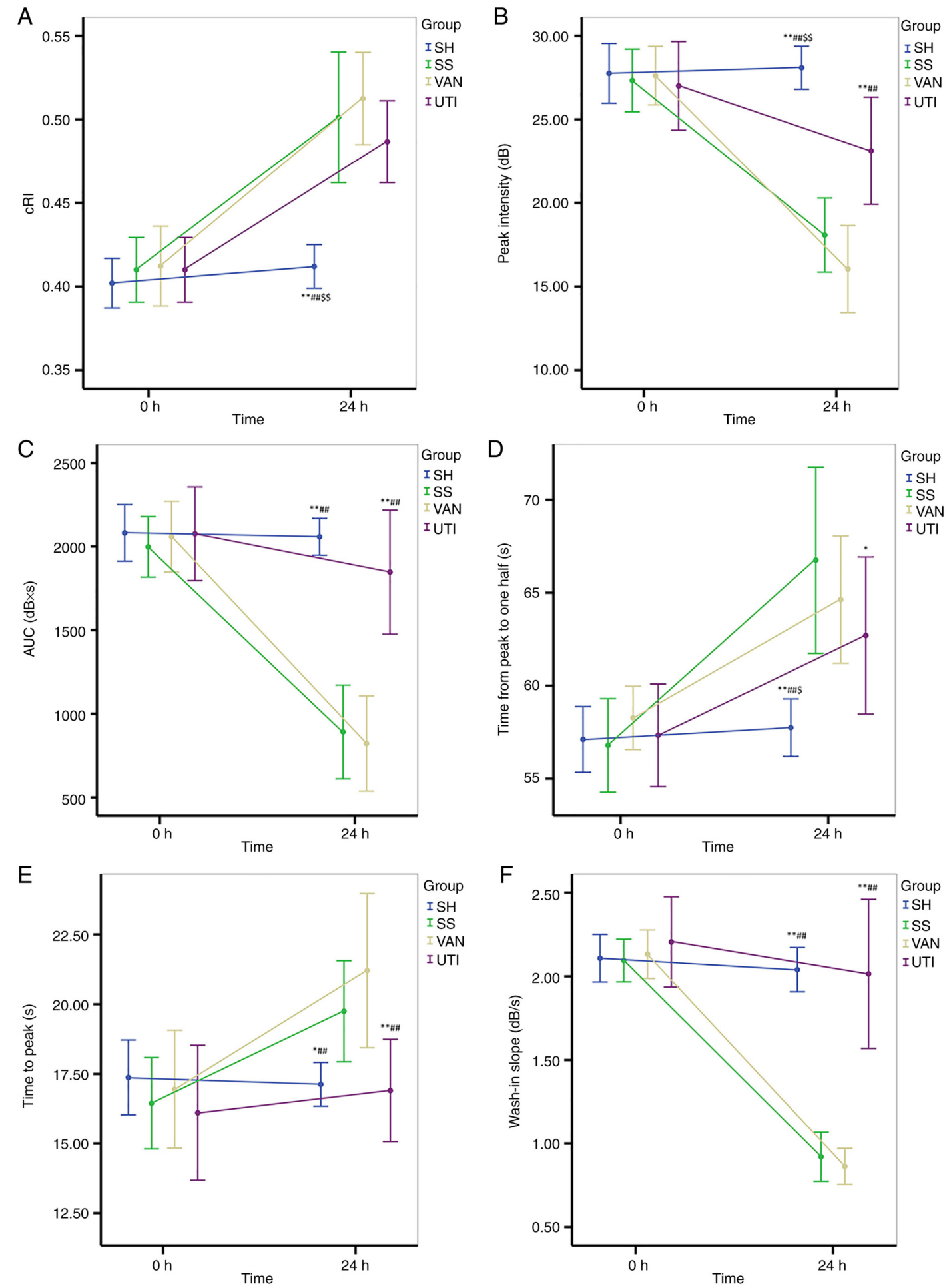

Figure 6. CDFI and CEUS. (A) cRI increased significantly at the end of the protocol except for that in the SH group. (B) Peak intensity decreased significantly after injury in all experimental groups. The improvement was higher in the UTI group than that in the SS and VAN groups. (C) AUC decreased after injury in the SS and VAN groups, but was improved in the UTI group. (D) Time from peak to 50\% of maximum and (E) time to peak significantly increased in the SS and VAN groups, but were improved by UTI treatment. (F) Wash-in slope significantly decreased in the SS and VAN groups, but was improved by UTI treatment. ${ }^{*} \mathrm{P}<0.05,{ }^{* *} \mathrm{P}<0.01$ vs. the SS group; ${ }^{\# \#} \mathrm{P}<0.01$ vs. the VAN group; ${ }^{\$} \mathrm{P}<0.05,{ }^{\$ \$} \mathrm{P}<0.01$ vs. the UTI group. CDFI, color doppler flow imaging; CEUS, contrast enhanced ultrasound; cRI, corrected resistive index; AUC, area under the curve. SH, sham group; SS, septic shock group; VAN, vancomycin group; UTI, ulinastatin group.

The Pi decreased significantly after injury in all experimental groups except the SH group, but was higher in the UTI group compared with that in the SS and VAN groups ( $\mathrm{P}<0.001$; Fig. 6B).

Similar to Pi, the AUC decreased after injury in the SS and VAN groups, but was improved in the UTI group (Fig. 6C).
Significant differences were identified between the UTI and SS or VAN groups, but were not found between the SH and UTI groups (Fig. 6C).

Compared with the baseline, the Th and Tp both increased significantly in the SS and VAN groups (Fig. 6D and E), 


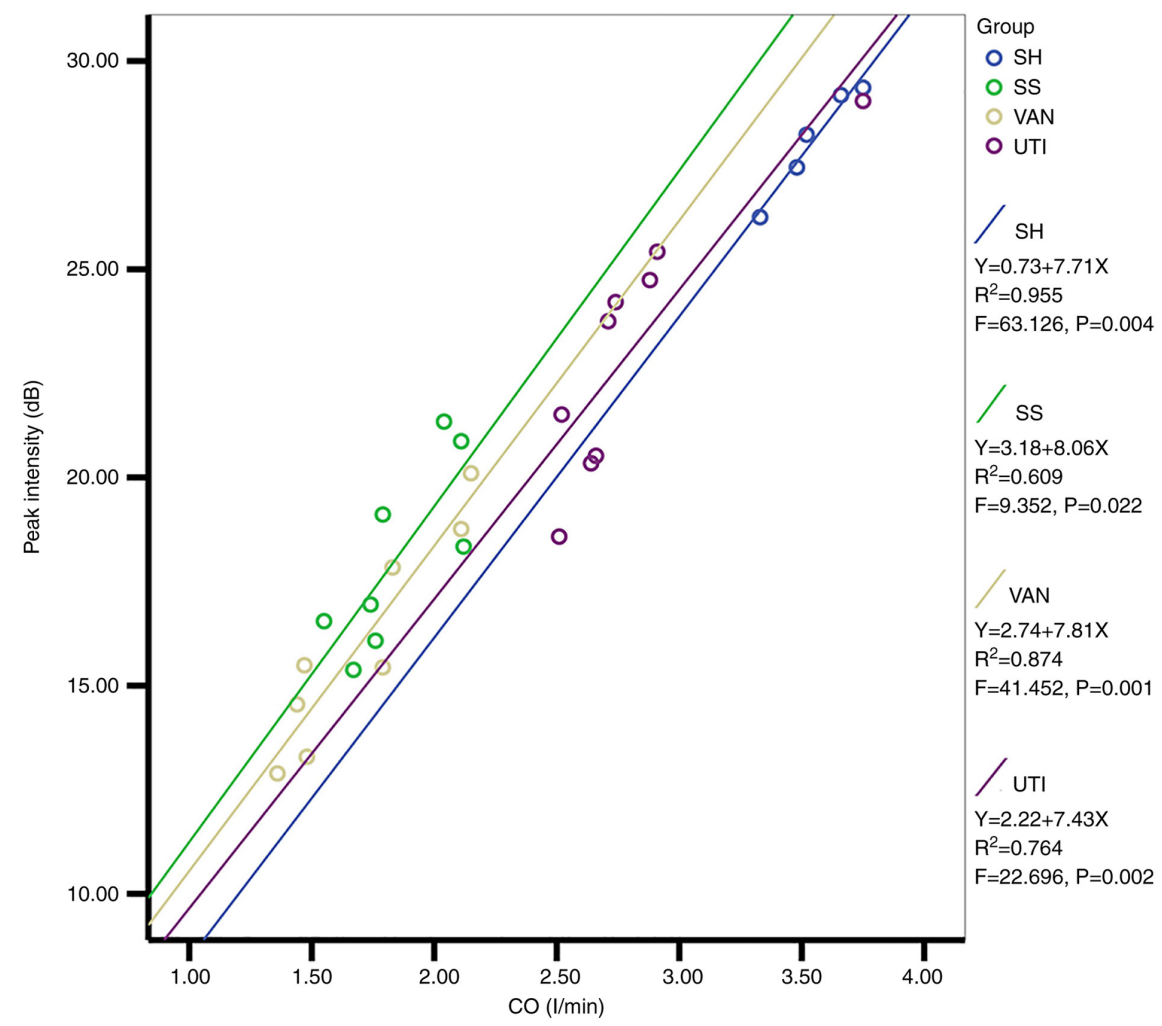

Figure 7. Linear regression analysis. Linear regression analysis between the $\mathrm{CO}$ and peak intensity in each group. There were similar positive regression coefficients in all four groups. CO, cardiac output; SH, sham group; SS, septic shock group; VAN, vancomycin group; UTI, ulinastatin group.

whereas the wash in slope decreased significantly. All of the parameters aforementioned were improved by the UTI treatment, where significant differences were found between the UTI and SS or VAN groups (Fig. 6D-F).

An association was identified by linear regression analysis between the $\mathrm{CO}$ and $\mathrm{Pi}$ in all groups (Fig. 7). The regression coefficients of the four equations were 7.71, 8.06, 7.81 and 7.43, respectively, and since they were similar, $\mathrm{CO}$ could be used as a covariate to perform the mixed two-way ANOVA again. Interestingly, the significant differences of $\mathrm{Pi}$ among the groups disappeared, which indicated that the decreased Pi in the SS, VAN and UTI group could be attributed to the decreased CO.

Renal histopathology. Compared with that in the SH group, renal injury was prominent in all experimental groups (Fig. 8A-D), especially in the mesenchyme. Glomerular capillary hyperemia, fibroblast proliferation near the glomerulus, micro-thrombus formation and diffusive inflammatory cell infiltration in the mesenchyme were observed. Under the LM, there was little to no injury in the $\mathrm{SH}$ group. The grade of renal injury was higher in the three experimental groups compared with that in the SH group, but it was lower in the UTI group compared with that in the SS and VAN groups (Table II). TUNEL assay revealed that there was higher AI in the SS and VAN groups than those in the SH and UTI groups where a significant difference was found between the UTI and SS or VAN groups (Fig. 8E-H; Table II).

Under TEM in the SS group, the epithelial degenerating chromatin was presented as nuclear cavitation, which demonstrated the cell apoptosis, whilst the mitochondria swelled, and the cristae disappeared (Fig. 9A and B).

\section{Discussion}

In total, 5-20\% patients who are critically ill experience an episode of AKI during the course of illness, which in many cases are accompanied with multiple organ dysfunction syndromes (20-22). A recent analysis from the PICARD Study Group observed AKI in patients who are critically ill, which is characterized by a large burden of comorbidity and can result in chronic kidney disease and extensive extra renal complications, necessitating dialysis (23). In the 21st century, a total of $\sim 4.9 \%$ patients in two years admitted to 30 intensive care units require renal replacement therapy in Austria (24). At the same time, a prospective hospital-based study in the UK found that the incidence of renal replacement therapy for AKI was 131 per million per year (25).

Renal perfusion can recover after sufficient fluid infusion, but microcirculation in the renal cortex cannot recover fully $(26,27)$. A fall in systemic blood pressure caused by hypovolemia activates the neurohumoral vasoconstrictive system to maintain blood pressure and cerebral perfusion, but not in the kidney (28). The autoregulation of kidney and blood pressure makes its perfusion stable within fairly narrow limits $(29,30)$. When MAP $>80 \mathrm{mmHg}$, this autoregulation can make the glomerular filtration rate (GFR) normal with perfusion; however, when the MAP reaches $<80 \mathrm{mmHg}$, the GFR decreases because of low perfusion $(29,30)$. In the present study model, MAP and the opposite changes of CO and SVR followed the hemodynamic features of septic shock. They changed in opposite directions: CO rapidly increased, whereas MAP and SVR decreased to the lowest point at $2 \mathrm{~h}$ after injury. From that time point, $\mathrm{CO}$ decreased whilst the SVR increased 
Table II. Histopathology outcomes.

\begin{tabular}{lcccc}
\hline Parameter & SH & SS & VAN & UTI \\
\hline Kidney injury scores & $0.0(0.5)^{\mathrm{a}-\mathrm{c}}$ & $3.0(0.0)$ & $3.0(0.0)$ & $2.0(1.0)^{\mathrm{d}, \mathrm{e}}$ \\
AI, \% & $1.50 \pm 0.23^{\mathrm{a}-\mathrm{c}}$ & $32.22 \pm 1.71$ & $30.11 \pm 2.67$ & $13.78 \pm 2.91^{\mathrm{a}, \mathrm{b}}$ \\
\hline
\end{tabular}

Kidney injury scores, median (IQR); AI, mean \pm SD. AI, apoptotic index; SH, sham group; SS, septic shock group; VAN, vancomycin group; UTI, ulinastatin group. ${ }^{a} \mathrm{P}<0.01$ vs. the SS group; ${ }^{b} \mathrm{P}<0.01$ vs. the VAN group and ${ }^{\mathrm{c}} \mathrm{P}<0.01$ vs. the UTI group; ${ }^{d} \mathrm{P}<0.05$ vs. the $\mathrm{SS}$ group; ${ }^{\text {eP }}<0.05$ vs. the VAN group.

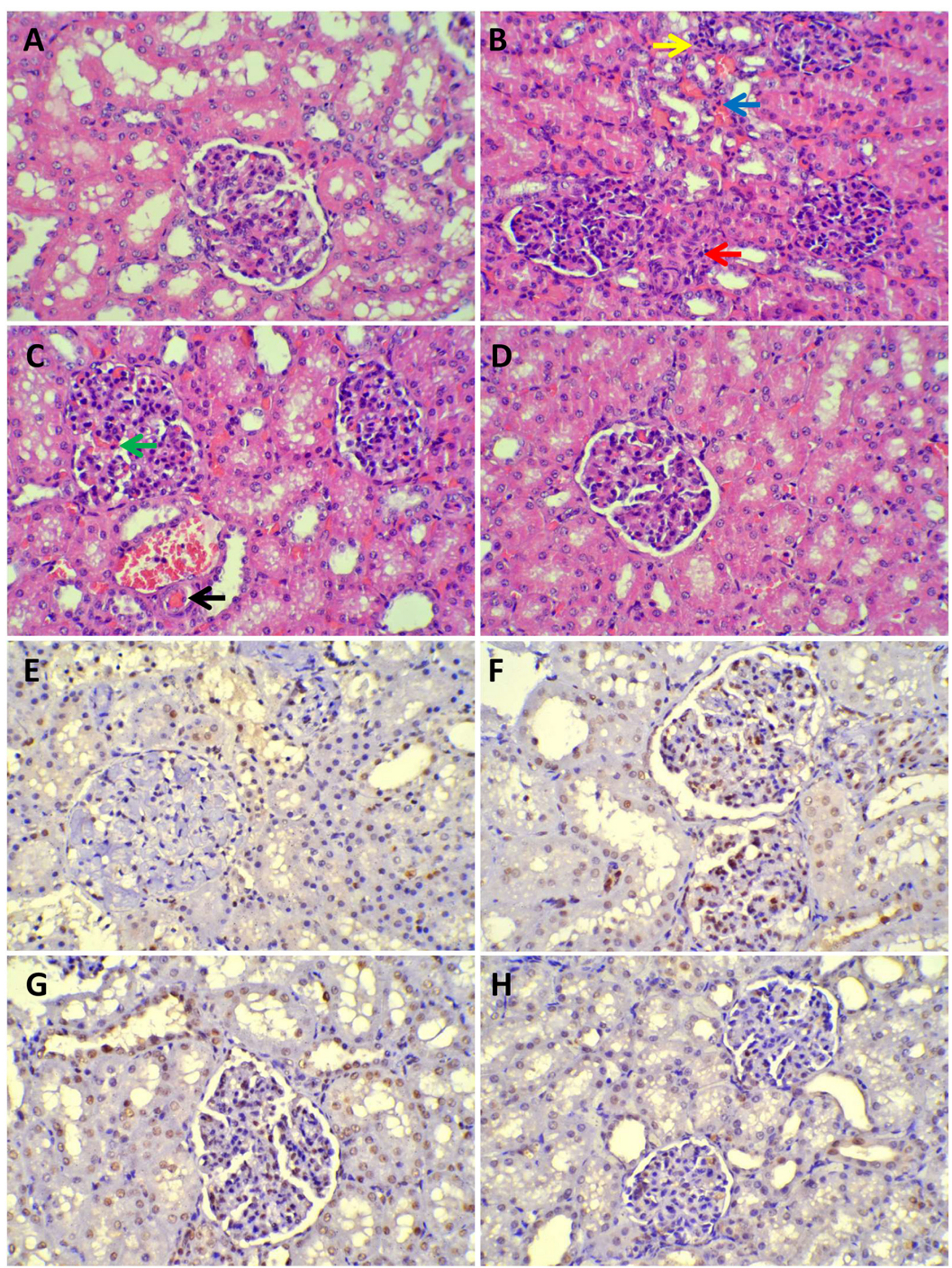

Figure 8. Histopathology of light microscope. (A-D) Under a light microscope, renal injury was prominent in all experimental groups except the sham group (magnification, x400). Red arrow, Fibroblast proliferation in the renal mesenchyme beside the glomerulus; yellow arrow, inflammatory cell infiltration; blue arrow, renal interstitial hyperemia; green arrow, glomerular capillaries angiectasis and hyperemia; black arrow, micro-thrombus formation. (A) Sham group. (B) SS group. (C) VAN group. (D) UTI group. (E-H) TUNEL assay revealed that there were greater numbers of apoptotic cells in the SS and VAN groups than the sham and UTI groups. Cells undergoing apoptotic signaling cascades were stained brown. (E) Sham group. (F) SS group. (G) VAN group. (H) UTI group. SS, septic shock group; VAN, vancomycin group; UTI, ulinastatin group.

gradually, whereas the MAP remained at a relatively low level in the range of 60-70 $\mathrm{mmHg}$. Since this model, developed in a previous study (12), displayed the typical hemodynamic features of septic shock, it was considered fit to be applied for the further investigation of intervention effects. The MAP of all modeling groups decreased $<80 \mathrm{mmHg}$ after injury, which could certainly make the renal perfusion decrease, and was confirmed by CEUS.

$\mathrm{RI}$ is a useful index for evaluating the renal vascular resistance in CDFI, which holds promise in monitoring renal function and in predicting AKI in patients who are critically ill (31). In the present study, cRI was calculated to correct for 

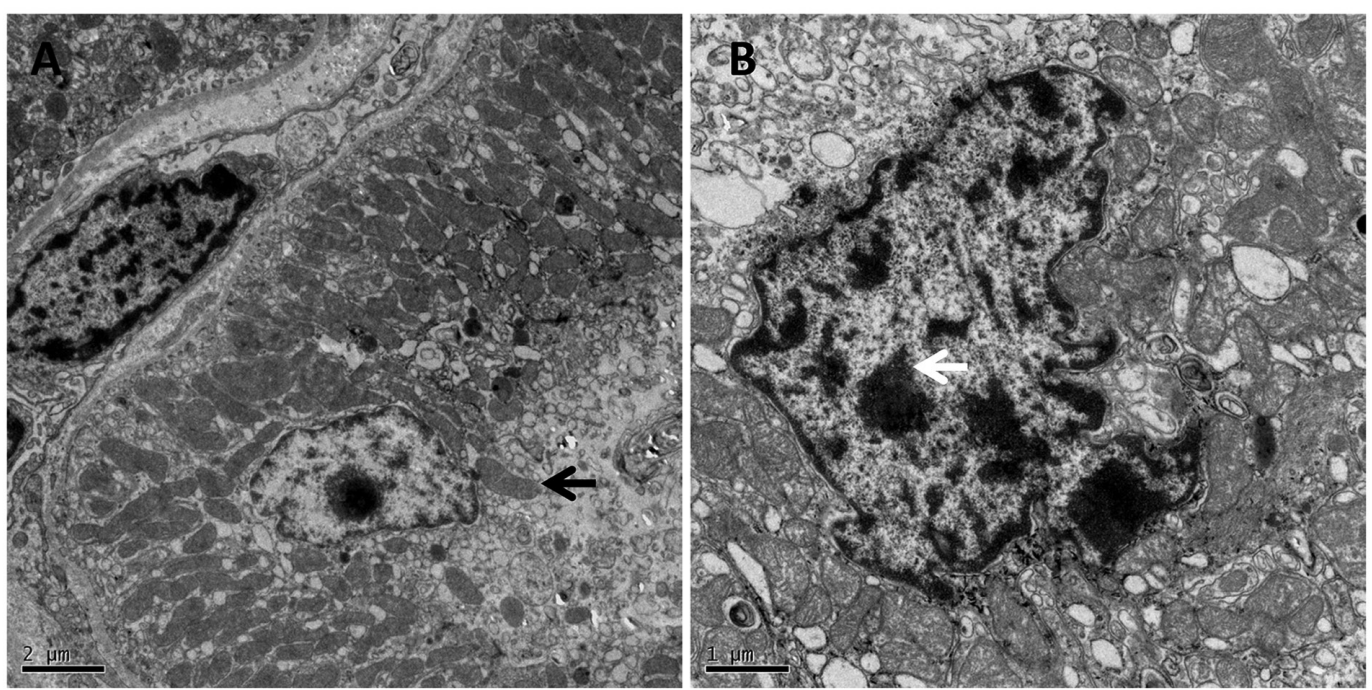

Figure 9. Histopathology of transmission electron microscope. Images of transmission electron microscope from the epithelia cells of the septic shock group. (A) Black arrow, the mitochondria swelled, and the cristae disappeared; (B) white arrow, chromatin degenerating indicated as the nuclear cavitation, which demonstrated the cell apoptosis.

the influence of HR and was increased in the present model, which indicated vasoconstriction in the kidney. A significant difference of cRI was not observed between the UTI group and SS or VAN group after UTI treatment, which demonstrated that UTI could not change the tension in the renal vascular system after septic shock. However, this did not necessarily mean that UTI had no involvement in regulating the renal microcirculation.

Dynamic contrast enhanced magnetic resonance imaging and isotopic renography are methods used to evaluate renal perfusion (32). However, the absolute quantification of renal perfusion using contrast enhanced magnetic resonance imaging is not reliable because of the poor association between signal intensity and concentration of the contrast medium (32). Isotopic renography can be used evaluate split renal function, but is limited by radiation exposure and high cost (33). CEUS had been previously proposed to be able to quantify renal cortical perfusion at bedside in the transplanted kidney. Microbubbles used in CEUS remain strictly intravascular, with little interstitial diffusion or urine excretion. They tend to produce more harmonic signals compared with surrounding tissues and are sensitive to the echo sound probe $(34,35)$. Due to the increased sensitivity of harmonic imaging, tissue perfusion on a capillary level can be detected using these media, that can be viewed as blood pool markers enabling functional vascular imaging (36). A good correlation has been demonstrated between CEUS measurements of renal perfusion and para-aminohippurate clearance, which is currently the gold standard for renal blood flow measurements (35). Previous data on animals also revealed that renal cortical CEUS can differentiate between macro- and microcirculation $(37,38)$. This technology has also been used in ICU for evaluating renal perfusion in two pilot studies $(39,40)$.

In the present study, TIC derived from CEUS was used to detect blood perfusion after septic shock, where the results indicated that the AUC was decreased significantly. AUC is a parameter related with the blood volume of the kidney (34). In the UTI group, it was significantly increased by the drug treatment compared with the SS and VAN group. No significant difference was observed between the SH and UTI group.

$\mathrm{Pi}$ is another parameter that is related to renal perfusion. In theory, if the same dose of microbubbles were bolus infused into the circulation and detected by the prober in the kidney, there should be the same Pi in each group (34). However, if $\mathrm{CO}$ decreased, only the time of reaching $\mathrm{Pi}$ would be delayed because of the low perfusion, but without the reduction in strength i.e. there would be an increased Tp with normal Pi (34). By contrast, if the $\mathrm{CO}$ was too low, before the whole dose of microbubbles filled the kidney, the washing out process would begin prematurely and the flow may decrease the Pi (34). In the present study, the Tp was significantly increased whereas the Pi was significantly decreased in the SS and VAN groups. Regression analysis between the $\mathrm{CO}$ and $\mathrm{Pi}$ indicated that there were similar regression coefficients in the four groups. Since $\mathrm{CO}$ was lower in the SS and VAN groups, it may decrease $\mathrm{Pi}$. Therefore, when $\mathrm{CO}$ was put into the ANOVA model as a covariate, the difference among the groups disappeared, which suggests that a lower $\mathrm{CO}$ cannot drive enough microbubbles into the renal cortex, thereby decreasing Pi. In the UTI group, both the Pi and Tp were significantly improved compared with that in the SS and VAN group.

The Th and slope are parameters related to the wash-in speed of microbubbles (34). During septic shock, both are deteriorated with poor perfusion. In the present study, the Th increased whereas the slope decreased, both of which were improved by UTI treatment. Unlike cRI, which is a parameter related to vascular tension, AUC, $\mathrm{Pi}$, Th and Slope, parameters of perfusion, were improved by UTI treatment, which demonstrated that the protective effect of UTI was not through a change in vascular resistance, but by the improvement of bloodflow. Oxygenation $\left(\mathrm{PaO}_{2} / \mathrm{FiO}_{2}\right)$ was also improved by UTI treatment together with the improved renal perfusion. In addition, the global aerobic metabolism was improved by UTI, as suggested by the observation of decreasing Lac levels.

Histopathological analysis confirmed the extent of kidney injury caused by septic shock and the subsequent 
improvement mediated by UTI treatment. Previous investigations indicated that $35-50 \%$ cases of acute tubular necrosis could be attributed to sepsis $(41,42)$. In the present model, tubular necrosis was not observed, which was likely to be due to the relatively short duration of the protocol. However, significant injury in the interstitium was found in the SS and VAN groups under LM. These injuries may progress to tubular necrosis after several weeks of sepsis $(41,42)$. Under EM, the cell injury observed were mitochondria swelling and chromatin degeneration, possibly contributing to apoptosis. Mitochondrial dysfunction has been previously described in organs with high metabolism, such as the kidney (43). TUNEL analysis also confirmed apoptosis in the renal cells. This histopathologic injury was ameliorated by UTI treatment, which may be due to improvements in renal perfusion and global aerobic metabolism.

In the present study, a phenomenon of improved renal perfusion by UTI was observed. Its macro-mechanism was confirmed by hemodynamic studies, though an animal model may not be the same as that in the clinical setting in humans. As a limitation of the present study, the micro-mechanism was not explored. Blood flow to the kidney is pronounced as the kidneys receive $\sim 25 \%$ of the total abdominal aortic blood flow and filter $120-150 \mathrm{ml}$ plasma per min, rendering it susceptible to the first attack by cytokines during inflammation. As a typical Kuniz protease inhibitor, UTI was previously demonstrated to regulate the expression of IL- 6 , IL- $1 \beta$ and TNF- $\alpha$ through the NF- $\kappa$ B pathway (44). Further studies should be performed surrounding the changes in level of cytokines regulated by the $\mathrm{NF}-\kappa \mathrm{B}$ protein pathway.

In conclusion, AKI, which occasionally occurs during septic shock, is accompanied with a significantly reduced perfusion in the renal microcirculation. However, UTI can significantly improve renal perfusion, which can be reliably evaluated using CEUS.

\section{Acknowledgements}

Not applicable.

\section{Funding}

The present study was funded by The Planning Project of Beijing Municipal Administration of Traditional Chinese Medicine (grant no. JJ2018-52).

\section{Availability of data and materials}

The datasets used and/or analyzed during the current study are available from the corresponding author on reasonable request.

\section{Authors' contributions}

$\mathrm{CCH}$ performed the experiments and drafted the manuscript. YHG acquired and analyzed data. CSL participated in the conception of the study and approved the final manuscript. SW designed the study and interpreted the results. YHG and SW confirm the authenticity of all the raw data. All authors have read and approved the final manuscript.

\section{Ethics approval and consent to participate}

The present study was approved by the Animal Care and Use Committee of Beijing Chao-Yang Hospital, Capital Medical University (Beijing, China), and the use of all animals that received treatment was in compliance with the National Research Council's 1996 Guide for the Care and Use of Laboratory Animals.

\section{Patient consent for publication}

Not applicable.

\section{Competing interests}

The authors declare that they have no competing interests.

\section{References}

1. Schrier RW and Wang W: Acute renal failure and sepsis. N Engl J Med 351: 159-169, 2004

2. Boffa JJ and Trendshorst WJ: Maintenance of renal vascular reactivity contributes to acute renal failure during endotoxemic shock. J Am Soc Nephrol 16: 117-124, 2005.

3. Schnell D and Darmon M: Renal Doppler to assess renal perfusion in the critically ill: A reappraisal. Intensive Care Med 38: 1751-1760, 2012.

4. Schnell D, Camous L, Guyomarc'h S, Duranteau J, Canet E, Gery P, Dumenil AS, Zeni F, Azoulay E and Darmon M: Renal perfusion assessment by renal Doppler during fluid challenge in sepsis. Crit Care Med 41: 1214-1220, 2013.

5. Puqia MJ, Valdes R Jr and Jortani SA: Bikunin (urinary trypsin inhibitor): Structure, biological relevance, and measurement. Adv Clin Chem 44: 223-245, 2007.

6. Inoue KI, Takano H, Yanagisawa R, Sakurai M, Shimada A, Yoshino S, Sato $\mathrm{H}$ and Yoshikawa T: Protective role of urinary trypsin inhibitor in acute lung injury induced by lipopolysaccharide. Exp Biol Med (Maywood) 230: 281-287, 2005.

7. Yang Q, Liu X, Liu M,Zhang L and Guan Y: Ulinastatin-mediated protection against zymosan-induced multiple organ dysfunction in rats. Biologicals 38: 552-556, 2010.

8. Ogawa M, Nishibe S, Mori T and Neumann S: Effect of human urinary trypsin inhibitor on granulocyte elastase activity. Res Commun Chem Pathol Pharmacol 55: 271-274, 1987.

9. National Research Council (US) Institute for Laboratory Animal Research: Guide for the care and use of laboratory animals. Washington (DC): National Academies Press (US), 1996.

10. Committee for the Update of the Guide for the Care and Use of Laboratory Animals. Guide for the care and use of laboratory animals. Washington, DC: National Research Council, National Academy Press, 2011.

11. Riebold TW, Geiser DR and Goble DO: General principles. In General Principles in Large Animal Anesthesia, Principles and Techniques, ed 2. Ames, 1A, Iowa State University Press, 1995

12. Wang S, Wang JY, Wang T, Hang CC, Shao R and Li CS: A novel porcine model of septic shock induced by acute respiratory distress syndrome due to methicillin-resistant staphylococcus aureus. Chin Med J (Engl) 130: 1226-1235, 2017.

13. Murakami K, Bjertnaes LJ, Schmalstieg FC, McGuire R, Cox RA, Hawkins HK, Herndon DN, Traber LD and Traber DL: A novel animal model of sepsis after acute lung injury in sheep. Crit Care Med 30: 2083-2090, 2002.

14. Enkhbaatar P, Joncam C, Traber L, Nakano Y, Wang J, Lange M, Connelly R, Kulp G, Saunders F, Huda R, et al: Novel ovine model of methicillin-resistant Staphylococcus aureus-induced pneumonia and sepsis. Shock 29: 642-649, 2008.

15. Jonkam CC, Bansal K, Traber DL, Hamahata A, Maybauer MO, Maybauer DM, Cox RA, Lange M, Connelly RL, Traber LD, et al: Pulmonary vascular permeability changes in an ovine model of methicillin-resistant Staphylococcus aureus sepsis. Crit Care 13: R19, 2009.

16. Leary S, Underwood W, Anthony R, Cartner S, Corey D, Grandin T, Greenacre CB, Gwaltney-Bran S, McCrackin MA, Meyer R, et al: AVMA guidelines for the euthanasia of animals: 2013 edition. American Veterinary Medical Association, Schaumberg, IL, 2013. 
17. Von Spiegel T, Wietasch G, Bürsch J and Hoeft A: Cardiac output determination with transpulmonary thermodilution: An alternative to pulmonary artery catheterization? Anaesthetist 45: 1045-1050, 1996 (In German).

18. Hershey BJ, Hagart JL and Havas KA: Clinical indicators of moribundity in swine experimentally inoculated with African swine fever virus. J Am Assoc Lab Anim Sci 60: 96-102, 2021.

19. Komatsu K, Frohlich ED, Ono H, Ono Y, Numabe A and Willis GW: Glomerular dynamics and morphology of aged spontaneously hypertensive rats. Effects of angiotensin-converting enzyme inhibition. Hypertension 25: 207-213, 1995.

20. Brivet FG, Kleinknecht DJ, Loirat $P$ and Landais PJ: Acute renal failure in intensive care units: Causes, outcome, and prognostic factors of hospital mortality: A prospective, multicenter study. Crit Care Med 24: 192-198, 1996.

21. de Mendonca A, Vincent JL, Suter PM, Moreno R, Dearden NM, Antonelli M, Takala J, Sprung C and Cantraine F: Acute renal failure in the ICU: Risk factors and outcome evaluated by the SOFA score. Intensive Care Med 26: 915-921, 2000

22. Liano F, Junco E, Pascual J, Madero R and Verde E: The spectrum of acute renal failure in the intensive care unit compared with that seen in other settings. Kidney Int Suppl 66: S16-S24, 1998.

23. Mehta RL, Pascual MT, Soroko S, Savage BR, Himmelfarb J, Ikizler TA, Paganini EP, Chertow GM and Program to Improve Care in Acute Renal Disease: Spectrum of acute renal failure in the intensive care unit: The PICARD experience. Kidney Int 66: 1613-1621, 2004.

24. Metnitz PG, Krenn CG, Steltzer H, Lang T, Ploder J, Lenz K, Le Gall JR and Druml W: Effect of acute renal failure requiring renal replacement therapy on outcome in critically ill patients. Crit Care Med 30: 2051-2058, 2002.

25. Metcalfe W, Simpson M, Khan IH, Prescott GJ, Simpson K, Smith WC, MacLeod AM and Scottish Renal Registry: Acute renal failure requiring renal replacement therapy: Incidence and outcome. QJM 95: 579-583, 2002.

26. Legrand M, Bezemer R, Kandil A, Demirci C, Payen D and Ince $C$ : The role of renal hypoperfusion in development of renal microcirculatory dysfunction in endotoxemic rats. Intensive Care Med 37: 1534-1542, 2011

27. Evans RG, Ince C, Joles JA, Smith DW, May CN, O'Connor PM and Gardiner BS: Haemodynamic influences on kidney oxygenation: Clinical implications of integrative physiology. Clin Exp Pharmacol Physiol 40: 106-122, 2013.

28. Badr KF and Ichikawa I: Prerenal failure: A deleterious shift from renal compensation to decompensation. N Engl J Med 319 623-629, 1988

29. Legrand $M$ and Payen D: Understanding urine output in critically ill patients. Ann Intensiva Care 1: 13, 2011

30. A Mitrou NG and Gupples WA: Renal blood flow dynamics in inbred rat strains provides insight into autoregulation. Curr Vasc Pharmacl 12: 801-809, 2014

31. Lerolle N, Guérot E, Faisy C, Bornstain C, Diehl JL and Fagon JY: Renal failure in septic shock: Predictive value of doppler-based renal arterial resistive index. Intensive Care Med 32: 1553-1559, 2006

32. Bokacheva L, Rusinek H, Zhang JL and Lee VS: Assessment of renal function with dynamic contrast-enhanced MR imaging. Magn Reson Imaging Clin N Am 16: 597-611, 2008.
33. El-Diasty MT, Gaballa G, Gad HM, Borg MA, Abou-Elghar ME, Sheir KZ and El-Diasty TA: Evaluation of CT perfusion parameters for assessment of split renal function in healthy donors. Egypt J Radiol Nucl Med 47: 1681-1688, 2016.

34. Hosotani Y, Takahashi N, Kiyomoto H, Ohmori K, Hitomi H, Fujioka H, Aki Y, Fukunaga M, Yuasa S, Mizushige K and Kohno M: A new method for evaluation of split renal cortical blood flow with contrast echography. Hypertens Res 25: 77-83, 2002.

35. Schneider AG, Hofmann L, Wuerzner G, Glatz N, Maillard M, Meuwly JY, Eggimann P, Burnier M and Vogt B: Renal perfusion evaluation with contrast-enhanced ultrasonography. Nephrol Dial Transplant 27: 674-681, 2012.

36. Blomley MJ, Albrecht T, Cosgrove DO, Jayaram V, Eckersley RJ, Patel N, Taylor-Robinson S, Bauer A and Schlief R: Liver vascular transit time analyzed with dynamic hepatic venography with bolus injections of an US contrast agent: Early experience in seven patients with metastases. Radiology 209: 862-866, 1998

37. Schlosser T, Pohl C, Veltmann C, Lohmaier S, Goenechea J, Ehlgen A, Köster J, Bimmel D, Kuntz-Hehner S, Becher H and Tiemann K: Feasibility of the flash-replenishment concept in renal tissue: Which parameters affect the assessment of the contrast replenishment? Ultrasound Med Biol 27: 937-944, 2001.

38. Wei K, Le E, Bin JP, Coggins M, Thorpe J and Kaul S Quantification of renal blood flow with contrast-enhanced ultrasound. J Am Coll Cardiol 37: 1135-1140, 2001.

39. Schneider AG, Goodwin MD, Schelleman A, Bailey M, Johnson L and Bellomo R: Contrast-enhanced ultrasound to evaluate changes in renal cortical perfusion around cardiac surgery: A pilot study. Crit Care 17: R138, 2013.

40. Schneider AG, Goodwin MD, Schelleman A, Bailey M, Johnson L and Bellomo R: Contrast-enhanced ultrasonography to evaluate changes in renal cortical microcirculation induced by noradrenaline: A pilot study. Crit Care 18: 653, 2014.

41. Cole L, Bellomo R, Silvester W and Reeves JH: A prospective, multicenter study of the epidemiology, management, and outcome of severe acute renal failure in 'closed' ICU system. Am J Respir Crit Care Med 162: 191-196, 2000.

42. Hoste EA, Lameire NH, Vanholder RC, Benoit DD, Decruyenaere JM and Colardyn FA: Acute renal failure in patients with sepsis in a surgical ICU: Predictive factors, incidence, comorbidity, and outcome. J Am Soc Nephrol 14: 1022-1030, 2003.

43. Nourbakhsh N and Singh P: Role of renal oxygenation and mitochondrial function in the pathophysiology of acute kidney injury. Nephron Clin Pract 127: 149-152, 2014.

44. Li W, Qiu X, Jiang H, Zhi Y, Fu J and Liu J: Ulinastatin inhibits the inflammation of LPS-induced acute lung injury in mice via regulation of AMPK/ NF- $\mathrm{BB}$ pathway. Int Immunopharmacol 29: $560-567,2015$

This work is licensed under a Creative Commons Attribution-NonCommercial-NoDerivatives 4.0 International (CC BY-NC-ND 4.0) License. 